\title{
A Case of Decreased Susceptibility to Ceftriaxone in Neisseria gonorrhoeae in the Absence of a Mosaic Penicillin-Binding Protein 2 (penA) Allele
}

\author{
A. Jeanine Abrams, PhD*, Robert D. Kirkcaldy, MD, MPH${ }^{\star}$, Kevin Pettus, BS ${ }^{*}$, Jan L. Fox, \\ MPH, RN ${ }^{\dagger}$, Grace Kubin, PhD ${ }^{\ddagger}$, David L. Trees, PhD $^{*}$ \\ *CDC Division of STD Prevention, Atlanta, GA; \\ †Oklahoma State Department of Health, HIV/STD Service, Oklahoma City, OK; \\ ¥Texas Department of State Health Services, Laboratory Services Section, Austin, TX
}

\section{Abstract}

We report a case of Neisseria gonorrhoeae with a non-mosaic penA allele that exhibited decreased susceptibility to extended-spectrum cephalosporins, including a ceftriaxone minimum inhibitory concentration of $0.5 \mu \mathrm{g} / \mathrm{mL}$. An analysis of resistance determinants suggested that the observed phenotype might have resulted from the combined effects of mutations in multiple genes.

Neisseria gonorrhoeae is the causative agent of the sexually transmitted disease (STD) gonorrhea, which is the second most commonly reported notifiable disease in the United States. ${ }^{1}$ Gonococcal infections are significant concerns due to the emergence of resistance to multiple antibiotics, which has been facilitated by both plasmid- and chromosome-mediated mechanisms. ${ }^{2}$ As a result of this acquired resistance, particularly to extended-spectrum cephalosporins (ESCs), the Centers for Disease Control and Prevention currently recommends dual therapy with ceftriaxone plus azithromycin as treatment for uncomplicated gonorrhea, ${ }^{3}$ and the treatment has been effective in the United States. Between 1987 and 2015, 5 isolates exhibiting decreased ceftriaxone susceptibility (minimum inhibitory concentration $[\mathrm{MIC}]=0.5 \mu \mathrm{g} / \mathrm{mL}$ ) were reported in the United States via the Gonococcal Isolate Surveillance Project (GISP) (https://www.cdc.gov/std/gisp/), ${ }_{1}$ including the isolate described in this case report.

In February 2012, a 32-year-old African-American male with symptoms of urethritis visited an STD clinic in Oklahoma City, Oklahoma that participated in GISP at that time. He reported recent sexual contact with a single female partner and denied prior gonococcal infection, current human immunodeficiency virus infection, sex with men, involvement with

Correspondence: A. Jeanine Abrams, PhD, Centers for Disease Control and Prevention, Division of STD Prevention, Laboratory Reference and Research Branch 1600 Clifton Rd. NE MS A12 Atlanta, GA 30333.xxp7@cdc.gov.

Publisher's Disclaimer: Disclaimer: The findings and conclusions in this report are those of the author(s) and do not necessarily represent the official position of the Centers for Disease Control and Prevention. 
the commercial sex trade, or recent travel. However, his female partner reported that she recently had sexual contact with a partner in Chicago, Illinois.

A urethral culture was obtained for inclusion in the GISP collection, and it tested positive for $N$. gonorrhoeae. The patient was treated with $250-\mathrm{mg}$ ceftriaxone as a single intramuscular injection, and he was prescribed 100-mg doxycycline to be taken orally twice daily for 1 week, based on the recommended treatment guidelines at that time. The asymptomatic female partner was located, and her urine sample tested positive for $N$. gonorrhoeae using a gonococcal-specific nucleic acid amplification test. The sample was tested using the BD Viper System (BD, New Jersey) in extracted mode using strand displacement amplification. A throat culture obtained from the female partner was negative for $N$. gonorrhoeae. She was also treated with a combination of ceftriaxone and doxycycline.

The urethral culture from the patient was subjected to antimicrobial susceptibility testing at a GISP reference laboratory at the Texas Department of State Health Services to determine the MICs for target antibiotics. Agar dilution testing revealed decreased susceptibility to the following ESCs: ceftriaxone (MIC $=0.5 \mu \mathrm{g} / \mathrm{mL}$ ), cefixime $(\mathrm{MIC}=1.0 \mu \mathrm{g} / \mathrm{mL}$ ), and cefpodoxime ( $\mathrm{MIC}=2.0 \mu \mathrm{g} / \mathrm{mL}$ ). The isolate was also resistant to penicillin ( $\mathrm{MIC}=2.0 \mu \mathrm{g} /$ $\mathrm{mL}$ ), and the tetracycline MIC value (MIC $=1.0 \mu \mathrm{g} / \mathrm{mL}$ ) was one dilution from the Clinical and Laboratory Standards Institute breakpoint of $2 \mu \mathrm{g} / \mathrm{mL}$ for resistance. The isolate exhibited susceptibility to both azithromycin (MIC $=0.5 \mu \mathrm{g} / \mathrm{mL}$ ) and ciprofloxacin (MIC $=$ $0.015 \mu \mathrm{g} / \mathrm{mL}$ ). These results were confirmed via agar dilution at the Centers for Disease Control and Prevention. The Oklahoma City-County Health Department STD program was notified of the results, and a health alert was issued to local healthcare providers on June 21, 2012. Numerous unsuccessful attempts were made by the STD program to locate the patient and his primary partner after the initial visit.

To further characterize the isolate (hereafter referred to as isolate 12CFX_T_009), wholegenome sequencing was used to identify resistance determinants associated with decreased susceptibility to cefixime and other extended-spectrum cephalosporins. Whole-genome sequencing was conducted using both short-read (Illumina HiSeq 2500 platform; European Nucleotide Archive accession number SAMEA3165247) and long-read (PacBio RSII platform) techniques. Regarding short-read data, de novo assembly and variant calling were conducted using SPAdes ${ }^{4}$ and CLC Genomics Workbench 9.0 software (www.qiagenbioinformatics.com), respectively, and the complete genome of gonococcal strain FA19 (Genbank accession no. CP012026) was used as a reference for variant calling. De novo assembly of long-read sequence data was conducted using the hierarchical genome assembly process workflow, which included consensus polishing using Quiver. ${ }^{5}$ To avoid variants due to sequencing errors, the short-read data were mapped to the assembled longread data (assembled as a single contig), and variant calling was conducted using CLC Genomics Workbench 9.0 software.

The following known gonococcal resistance determinants were examined to identify mutations potentially associated with reduced susceptibility to $\beta$-lactam antibiotics and ESCs: penicillin-binding protein 2 (penA), penicillin-binding protein 1 (ponA), multiple transferable resistance repressor $(m t r R)$, porin B (por $B)$, and type IV pilus biogenesis and 
competence protein PilQ (pilQ) ${ }^{6,7}$ In addition, both major pilin subunit (pilE) and type IV pilus tip-located adhesion PilC ( $p$ ilC1 and pilC2) genes were examined because the products of these genes interact with the pilQ product during pilus formation. ${ }^{8,9}$

The results of the comparison of the short- and long-read sequence data indicated an error rate of $0.01 \%$ between the 2 methods, and no discrepancies were found between sequences of the target genes. The results of $N$. gonorrhoeae multiantigen sequence typing and multilocus sequence typing analyses identified the strain as ST14647 and ST1893, respectively. Variation was detected in each of the target resistance genes (Table 1). Mosaic pen $A$ alleles are strongly associated with reduced susceptibility to cefixime and ceftriaxone. ${ }^{10}$ However, the results indicated that the pen $A$ allele for isolate 12CFX_T_009 was a nonmosaic pen $A$ IX allele, which has not been associated with reduced susceptibility to ESCs. Regarding pon $A$, a single mutation (421P) associated with reduced susceptibility to cephalosporins was identified. ${ }^{6}$ However, this particular pon $A$ mutation has not been linked to ceftriaxone MIC values greater than $0.125 \mu \mathrm{g} / \mathrm{mL} .{ }^{11}$ Mutations in the $m t r R$ promoter (a single adenine deletion) and the $m t r R$ coding region (45D) can lead to increased expression of the MtrCDE efflux pump, which could lead to reduced susceptibility to ESCs. 6,12 Although the 45D mutation was detected (in the absence of the promoter mutation), it has not been associated with ceftriaxone MIC values comparable to that observed in this case. por $B$ exhibited extensive variability, but only mutations at residues 120 and 121 in this gene have been associated with resistance to $\beta$-lactam antibiotics. ${ }^{13}$ However, the por $B$ mutations identified in this case $(120 \mathrm{~N}$ and $121 \mathrm{~A})$ have not previously been associated with resistance to $\beta$-lactam antibiotics. Regarding pilQ, the $666 \mathrm{~K}$ mutation is associated with resistance to penicillin based on in vitro experimentation, ${ }^{14}$ but this mutation has not been identified in clinical samples. ${ }^{15}$ The $666 \mathrm{~K}$ mutation was not found is this case, and the 3 identified mutations have not been associated with resistance to $\beta$-lactam antibiotics in clinical isolates. The greatest variability was observed in pilE, pilC1, and pilC2, which contained 14, 198, and 366 missense mutations, respectively. Although specific mutations in these genes are not associated with resistance, the products of these genes interact with the pilQ product during pilin synthesis; therefore, drastic mutations (e.g., nonsense mutations resulting in nonfunctional or truncated products) could affect the latter. However, no early stop codons were identified during examinations of the transcribed amino acid sequences of these 3 genes.

Although a strong association between mosaic pen $A$ alleles and increased resistance to ESCs in $N$. gonorrhoeae has been established,${ }^{10}$ reduced susceptibility to ESCs has also been detected in isolates containing non-mosaic penA alleles. ${ }^{7,16,17}$ Although the isolate described in this case exhibited a non-mosaic pen $A$ allele in conjunction with several mutations in other resistance loci (pon $A, m t r R$, por B, and pilQ), none of the identified mutations were previously individually associated with the observed elevated ceftriaxone MIC levels $(0.5 \mu \mathrm{g} / \mathrm{mL})$. Therefore, the results of this analysis suggest that in the absence of major mutations, the observed reduced susceptibility might be due to a multilocus phenomenon resulting from the combined effects of mutations at multiple targets or via unknown compensatory changes at secondary loci. ${ }^{18}$ Furthermore, these results suggest that molecular tests that aim to identify reduced susceptibility to ESCs in $N$. gonorrhoeae will have to take multiple mutations and mutational combinations into account to obtain accurate 
diagnoses. Future analyses of this and similar isolates warrant the investigation of these mechanisms, which could drastically impact the extent of antimicrobial resistance in $N$. gonorrhoeae.

\section{REFERENCES}

1. Centers for Disease Control and Prevention. Sexually Transmitted Disease Surveillance 2015. Atlanta. U.S. Department of Health and Human Services 2016.

2. Tapsall JW. Antimicrobial resistance in Neisseria gonorrhoeae. Geneva: World Health Organization, WHO Document CDS/CSR/DRS, 2001.

3. Workowski KA, Bolan GA. Centers for Disease Control and Prevention. Sexually Transmitted Diseases Treatment Guidelines, 2015. MMWR Recomm Rep 2015; 64:1-137.

4. Bankevich A, Nurk S, Antipov D, et al. SPAdes: A new genome assembly algorithm and its applications to single-cell sequencing. J Comput Biol 2012; 19:455-477. [PubMed: 22506599]

5. Chin CS, Alexander DH, Marks P, et al. Nonhybrid, finished microbial genome assemblies from long-read SMRT sequencing data. Nat Methods 2013; 10:563-569. [PubMed: 23644548]

6. Lindberg R, Fredlund H, Nicholas R, et al. Neisseria gonorrhoeae isolates with reduced susceptibility to cefixime and ceftriaxone: Association with genetic polymorphisms in penA, mtrR, porB1b, and ponA. Antimicrob Agents Chemother 2007; 51:2117-2122. [PubMed: 17420216]

7. Lee SG, Lee H, Jeong SH, et al. Various penA mutations together with mtrR, porB and ponA mutations in Neisseria gonorrhoeae isolates with reduced susceptibility to cefixime or ceftriaxone. $\mathrm{J}$ Antimicrob Chemo 2010; 65:669-675.

8. Drake SL, Sandstedt SA, Koomey M. PilP, a pilus biogenesis lipoprotein in Neisseria gonorrhoeae, affects expression of PilQ as a high-molecular-mass multimer. Mol Microbiol 1997; 23: 657-668. [PubMed: 9157238]

9. Craig L, Li J. Type IV pili: Paradoxes in form and function. Curr Opin Struct Biol 2008; 18:267277. [PubMed: 18249533]

10. Grad YH, Kirkcaldy RD, Trees D, et al. Genomic epidemiology of Neisseria gonorrhoeae with reduced susceptibility to cefixime in the USA: A Retrospective Observational Study. Lancet Infect Dis 2014; 14:220-226. [PubMed: 24462211]

11. Ohnishi M, Golparian D, Shimuta K, et al. Is Neisseria gonorrhoeae initiating a future era of untreatable gonorrhea?: Detailed characterization of the first strain with high-level resistance to ceftriaxone. Antimicrob Agents Chemother 2011; 55:3538-3545. [PubMed: 21576437]

12. Zarantonelli L, Borthagaray G, Lee EH, et al. Decreased azithromycin susceptibility of Neisseria gonorrhoeae due to mtrR mutations. Antimicrob Agents Chemother 1999; 43: 2468-2472. [PubMed: 10508026]

13. Olesky M, Hobbs M, Nicholas RA. Identification and analysis of amino acid mutations in porin IB that mediate intermediate-level resistance to penicillin and tetracycline in Neisseria gonorrhoeae. Antimicrob Agents Chemother 2002; 46:2811-2820. [PubMed: 12183233]

14. Zhao S, Tobiason DM, Hu M, et al. The penC mutation conferring antibiotic resistance in Neisseria gonorrhoeae arises from a mutation in the PilQ secretin that interferes with multimer stability. Mol Microbiol 2005; 57:1238-1251. [PubMed: 16101998]

15. Whiley DM, Jacobsson S, Tapsall JW, et al. Alterations of the pilQ gene in Neisseria gonorrhoeae are unlikely contributors to decreased susceptibility to ceftriaxone and cefixime in clinical gonococcal strains. J Antimicrob Chemother 2010; 65:2543-2547. [PubMed: 20940180]

16. Whiley DM, Limnios EA, Ray S, et al. Diversity of penA alterations and subtypes in Neisseria gonorrhoeae strains from Sydney, Australia, that are less susceptible to ceftriaxone. Antimicrob Agents Chemother 2007; 51:3111-3116. [PubMed: 17591846]

17. Osaka K, Takahata M, Takakura T, et al. Analysis of amino acid sequences of penicillin-binding protein 2 in clinical isolates of Neisseria gonorrhoeae with reduced susceptibility to cefixime and ceftriaxone. J Infect Chemother 2008; 14:195-203. [PubMed: 18574654]

18. Day T, Gandon S. The evolutionary epidemiology of multilocus drug resistance. Evolution 2012; 66:1582-1597. [PubMed: 22519792] 
TABLE 1.

Target Resistance Genes and Associated Mutations

\begin{tabular}{ll}
\hline Gene & \multicolumn{1}{c}{ Mutations $^{*}$} \\
\hline penA & ins346D, 505L, 511V, 517G, 542H, and 552L; non-mosaic IX \\
ponA & $421 \mathrm{P}$ \\
mtrR & $45 \mathrm{D}$ \\
porB & 80 missense including 120N and 121A \\
pilQ & $341 \mathrm{~N}, 523 \mathrm{G}$, and $648 \mathrm{~N}$ \\
pilC1 & 198 missense \\
pilC2 & 366 missense \\
pile & 14 missense \\
\hline$*$ &
\end{tabular}

